\title{
Percepción y Accesibilidad Tecnológica de Universitarios en el Suroeste de República Dominicana durante el Covid-19
}

\section{Perception and Technological Accessibility of University Students in Southwest of the Dominican Republic during Covid-19}

Leandro M. Santos *, Diana Grisales y José Suero Rico

Instituto Superior de Formación Docente Salomé Ureña, República Dominicana

\section{DESCRIPTORES:}

Aprendizaje en línea

Educación a distancia

Enseñanza superior

Formación de docentes

TIC

\section{RESUMEN:}

La pandemia generó suspensión de clases presenciales y la implementación de la educación virtual. El objetivo de este estudio fue diagnosticar y analizar la percepción de estudiantes universitarios y su accesibilidad tecnológica durante la emergencia educativa en el suroeste de República Dominicana (enero-abril de 2020). Se empleó un método mixto de investigación, se aplicó una encuesta y se analizaron las respuestas mediante estadística descriptiva e inferencia estadística, análisis descriptivo y construcción de redes semánticas. Respecto a la accesibilidad tecnológica, los resultados muestran que predomina el uso de teléfonos en vez de computadoras y falta de acceso a Internet. Según los estudiantes existen más aspectos negativos que positivos en la educación virtual, ya que faltan equipos tecnológicos y accesibilidad a internet, lo que genera frustración e incomodidad, así como dificultades de aprendizaje. También la sobrecarga de asignaturas y actividades, ya que más de 60 tareas fueron asignadas para desarrollar en un mes, lo que para ellos demuestra insensibilidad de los docentes frente a la situación. El distanciamiento social generó ansiedad debido al aislamiento y falta de concentración para el aprendizaje. Entre los aspectos positivos está el uso de la tecnología, que facilita el aprendizaje y permite la continuidad de los estudios.

\section{ABSTRACT:}

\section{Virtual learning}

Distance education

Higher education

Teacher training

ICT
The pandemic led to suspension of face-to-face classes and the implementation of virtual education. The objective of this study was to diagnose and analyze the perception of university students and their technological accessibility during the educational emergency in the southwest of the Dominican Republic (January-April 2020). A mixed research method was used, a survey was applied, and responses were analyzed using descriptive statistics and statistical inference, descriptive analysis, and semantic network construction. Regarding technological accessibility, the results show that the use of phones predominates rather than computers and lack of Internet access. According to students there are more negative aspects than positives in virtual education, as technological equipment and internet accessibility are lacking, resulting in frustration and discomfort, as well as learning difficulties. Also, the overload of subjects and activities, since more than 60 tasks were assigned to develop in a month, which for them shows insensitivity of teachers in the face of the situation. Social distancing generated anxiety due to isolation and lack of concentration for learning. Among the positive aspects is the use of technology, which facilitates learning and allows the continuity of studies.

CÓMO CITAR:

Santos, L. M., Grisales, D. y Suero Rico, J. (2021). Percepción y accesibilidad tecnológica de universitarios en el suroeste de rRepública Dominicana durante el Covid-19. Revista Internacional de Educación para la Justicia Social, 10(1), 145-165. https://doi.org/10.15366/riejs2021.10.1.009

${ }^{*}$ Contacto: leandro.mattos@isfodosu.edu.do

ISSN: 2254-3139

revistas.uam.es/riejs
Recibido: 5 de julio 2020

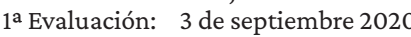

2a Evaluación: 9 de noviembre 2020

Aceptado: 24 de diciembre 2020 


\section{Introducción}

El día 11 de marzo de 2020, la Organización Mundial de la Salud pasó a caracterizar la Covid-19 como una pandemia. Una de las medidas sugeridas para controlar la propagación y contagio por el coronavirus (SARSCoV-2) fue el aislamiento social. Esto ocasionó el cierre de centros educativos y consecuentemente la suspensión de las clases presenciales, iniciando la emergencia remota educativa debido a la Covid-19. En el mundo, el 91\% de la población estudiantil fue afectada con la suspensión de clases (UNESCO, 2020a). Con la finalidad de mitigar los efectos de la interrupción de la educación presencial, la educación en línea fue y está siendo utilizada para dar continuidad a los procesos de enseñanza y aprendizaje (UNESCO, 2020b).

En América Latina y el Caribe, las Instituciones de Educación Superior y universidades, también decidieron suspender las clases y actividades presenciales (UNESCO-IESALC, 2020). En la República Dominicana (RD), más de 500,000 estudiantes universitarios fueron impactados con las medidas de aislamiento social y suspensión de clases (UNESCO, 2020a). Como respuesta, la mayoría de las instituciones implementaron la virtualización de clases, aunque con poca orientación previa sobre el desarrollo efectivo de los procesos de aprendizaje virtual utilizando las Tecnologías de la Información y Comunicación (TIC) (UNESCO-IESALC, 2020). Sin embargo, debido a cuestiones socioeconómicas, una parcela de la sociedad tiene acceso limitado al uso de las TIC.

La República Dominicana presenta un Índice de Desarrollo Humano (IDH) alto, ocupando el puesto 89 de 189 países evaluados, con Coeficiente de Gini (medida de desviación de la distribución de los ingresos entre los ingresos u hogares) igual a 45,7 (Programa de las Naciones Unidas para el Desarrollo [PNUD], 2019). No obstante, en el suroeste de RD, las provincias de Azua (región Valdesia), Bahoruco, Barahona, Independencia, Pedernales (región Enriquillo), Elías Piña y San Juan (región El Valle) presentan graves problemas socioeconómicos, con un IDH de bajo a medio bajo (Programa de las Naciones Unidas para el Desarrollo, República Dominicana [PNUD-RD], 2013, 2019). Además del IDH, otros parámetros socioeconómicos están representados en cifras y porcentajes siempre menores en el suroeste de RD comparado con el resto del país. Por ejemplo, el desempleo y la pobreza de los hogares en Valdesia es de 52,2\% y 24,2\%, en Enriquillo es de 5,8\% y $23,6 \%$, en El Valle es de $48,6 \%$ y $25,2 \%$, mientras la media en el país es de $44,6 \%$ y 17,2\% (Oficina Nacional de Estadística [ONE], 2018).

Otro aspecto que resaltar es la baja calidad de la educación en el país, demostrada en los resultados del Programa Internacional para la Evaluación de Estudiantes PISA (Programme for International Student Assessment). En esta evaluación, RD quedó en las últimas posiciones en Lectura, Matemática y Ciencias entre los países evaluados (Organisation for Economic Co-operation and Development [OECD], 2019). Al mismo tiempo, los estudiantes con ventajas socioeconómicas superaron en puntuación a los estudiantes con problemas económicos (OECD, 2019). Sin embargo, el promedio de este dato es inferior al de países miembros de la OECD (OECD, 2019).

Teniendo en cuenta las problemáticas socioeconómicas y educativas, además de las medidas de aislamiento social debido a la pandemia, que conllevó a la suspensión de clases presenciales y a la implementación de la educación en línea debido, es importante evaluar cómo la emergencia remota educativa afectó los estudiantes universitarios en el suroeste de RD.

\section{Revisión de la literatura}

La educación a distancia se desarrolló en tres fases o generaciones. La primera generación se caracterizó por el uso predominante de una sola tecnología y la falta de interacción directa entre estudiante y la institución educativa (Bates, 2005). La segunda generación de la educación a distancia se caracterizó por el uso de material impreso en conjunto con la radiodifusión. En esta, los materiales de aprendizaje fueron diseñados específicamente para el estudio a distancia, pero la comunicación con los estudiantes era mediada por un tutor y no por aquel que elaboró el material didáctico (Bates, 2005).

La educación a distancia en su tercera generación se basa en medios de comunicación bidireccionales, como el Internet o videoconferencias que permiten la interacción entre el profesor que origina la instrucción y el alumno remoto (Bates, 2005). Otro aspecto característico de la tercera generación es que el uso de Internet es considerado uno de los cambios más transformadores del contexto del proceso educativo, facilitando la 
comunicación entre los estudiantes y revolucionando la educación a distancia (Bates, 2005; Viñals y Cuenca, 2016).

La accesibilidad a Internet viene mejorando en América Latina y el Caribe, pero todavía constituye un problema para la implementación de la enseñanza virtual (Nye, 2015; Scagnoli, 2009). En RD, únicamente 30\% de los hogares dominicanos tienen Internet (ONE, 2018). En el suroeste del país la tasa de accesibilidad es más baja, como, en la región El Valle, donde solo 10,9\% de los hogares tienen internet, mientras la región Enriquillo 12,2\% y la región Valdesia 19,2\% (ONE, 2018). La media de velocidad de descarga de banda ancha en el país es de 22,8 Mbps, mediana 10,0 Mbps, mientras que en teléfonos móviles es de 25,1 Mbps, mediana 13,9 Mbps. Además del Internet, el uso de teléfonos, tabletas, computadores, aplicaciones y recursos multimedia son fundamentales para el desarrollo del aprendizaje y de la enseñanza en línea (Bates, 2015; Burns, 2011).

El porcentaje de la población de 5 años y más que posee computadora de escritorio, portátil y tableta es de 10,6\% en RD, mientras que teléfonos celulares es de 69,3\% (ONE, 2018). No obstante, en las regiones del suroeste del país la accesibilidad de equipos tecnológicos es menor. En la región del El Valle, 5,5\% de la población tiene computadoras, en Enriquillo 6,4\%, mientras en Valdesia 7,8\%. Con relación a la posesión de teléfonos, el porcentaje es de 56,1\% en El Valle, 54,2\% en Enriquillo y 63,9\% en Valdesia (ONE, 2018).

Para amenizar la brecha digital, el gobierno dominicano creó el programa República Digital con el objetivo de aumentar la accesibilidad a equipos tecnológicos y conectividad a internet (García et al., 2019). Uno de los proyectos, llamado "Una computadora para estudiantes y maestros de educación superior", estuvo dirigido a 18,000 estudiantes de las carreras de Educación en todo el país. Sin embargo, el alcance del programa con respecto al número de computadoras distribuidas para estudiantes no es mencionado, únicamente el número de estudiantes y maestros capacitados.

Según Bates (2005), la disminución de los costos para acceder a diversas herramientas tecnológicas es un factor que contribuye para el uso de las TIC en un contexto educativo, principalmente para los docentes. Sin embargo, una parte de la población no posee los recursos económicos para tener computadoras y acceso continuo al Internet. Consecuentemente, si los estudiantes no tienen acceso a la tecnología, no pueden estudiar y aprender bajo la modalidad a distancia (Bates, 2005, 2015; Ramas Arauz, 2015). Por consiguiente, la tecnología utilizada en la enseñanza a distancia debe soportar la educación (Bates, 2005, 2015; Burns, 2011) y no reforzar las desigualdades (Tait y O'Rourke, 2014). Al mismo tiempo, el aprendizaje a distancia debe considerar el acceso, la equidad y la entrega de un servicio educacional de calidad (Wright, Dhanarajan y Reju, 2009).

El acceso a hardware, la fiabilidad del Internet y del sistema de energía y los costos del servicio son consideradas las barreras para el uso de las TIC en la educación, principalmente en los países en desarrollo (Nye, 2015; Wright, Dhanarajan y Reju, 2009). Además, de la falta de accesibilidad a las TIC, los docentes pueden carecer de competencias digitales, debido a la inexperiencia, lo que genera desconfianza y/o inseguridad en el uso de computadoras para la enseñanza en línea (Bingimlas, 2009).

Los aspectos tecnológicos, como la accesibilidad al internet, equipos y la infraestructura para ofrecer el aprendizaje en línea constituyen un aspecto fundamental y discriminante para la educación a distancia, sin embargo, no es el único (Bates, 2005, 2015). Instituciones educativas, docentes y estudiantes también deben estar atentos a las peculiaridades de la educación a distancia.

Las instituciones educativas al considerar el uso del internet para la enseñanza deben obtener datos confiables acerca de la accesibilidad tecnológica y demografía de estudiantes y docentes con el objetivo de garantizar el acceso y la equidad educativa (Bates, 2005, 2015; Wright, Dhanarajan y Reju, 2009). Además, deben adaptar los currículos para aprovechar al máximo el uso de la tecnología, y promover una interacción significativa entre los estudiantes, el material de aprendizaje y los docentes (Wright, Dhanarajan y Reju, 2009). Al mismo tiempo, deben ofrecer capacitación a los docentes e instrucciones a los estudiantes con relación a tecnología y el proceso de enseñanza y aprendizaje en línea. Simultáneamente, deben ofrecer incentivos a la docencia virtual y evaluar constantemente los resultados de aprendizaje.

Considerando que la educación en línea es diferente de la presencial, para una enseñanza eficaz en educación superior, los docentes necesitan desempeñar diversas funciones y competencias que son realizadas mediante las TIC, además de considerar las diferencias de aprendizaje entre los estudiantes de forma remota (Bates, 2015; Coppola, Hiltz y Rotter, 2002; Ní Shé et al., 2019). Entre las diversas funciones citadas en la literatura, destacamos la afectiva, de gestión, pedagogía, social, técnica, evaluador, facilitador y experto en contenido 
(Burns, 2011; Coppola, Hiltz y Rotter, 2002; Muñoz, González y Hernández, 2013; Ní Shé et al., 2019; Oyarzun, Martin y Moore, 2020). La presencia, la facilitación y el apoyo a los estudiantes son considerados elementos primordiales para la docencia en línea (Bates, 2015; Dunlap y Lowenthal, 2018; Ní Shé et al., 2019).

Con relación al aprendizaje virtual, es necesario que los estudiantes desarrollen la autonomía, la responsabilidad, la curiosidad, la motivación, el aprendizaje autónomo, la autorregulación, el manejo del tiempo y de la información en el internet (Ally, 2008; Berridi Ramírez y Martínez Guerrero, 2017; Burns, 2011; Martin y Bolliger, 2018). Al mismo tiempo, los alumnos deben evitar la procrastinación (Cerezo et al., 2017). La accesibilidad tecnológica de los estudiantes, la preparación de los profesores y de la institución deberían ser las principales preocupaciones, pues el incumplimiento de estos genera dificultades para estudiantes y maestros y son barreras para el desarrollo eficaz de las clases virtuales (Bates, 2005, 2015; Burns, 2011).

Las consecuencias negativas de la emergencia remota educativa debido a la pandemia por Covid-19, son reveladas a cada día. Algunas de estas pueden ser la interrupción del aprendizaje, la tendencia al abandono escolar y el acceso desigual a las plataformas de aprendizaje digital, que afecta la igualdad y equidad educativa (Álvarez et al., 2020; Holguín y Sandoval, 2020). Además, se ha notado el aumento de la ansiedad debido a las medidas de aislamiento social en estudiantes y docentes (Cao et al., 2020).

Teniendo en cuenta las problemáticas socioeconómicas y educativas de la República Dominicana, así como el agravamiento de las desigualdades sociales debido a la situación actual de la pandemia y las acciones tomadas con el fin de minimizar los efectos de la suspensión de clases presenciales mediante cursos virtuales, esta investigación tuvo como objetivo diagnosticar la accesibilidad a herramientas tecnológicas y la competencia para su uso, así como la percepción de la situación de la emergencia remota educativa en estudiantes universitarios en el suroeste de la República Dominicana.

\section{Método}

En RD existen alrededor de 550,000 estudiantes universitarios. La institución en la cual fue realizada esta investigación es responsable por la formación docente en el país y cuenta con seis recintos en diferentes regiones y 3,839 estudiantes. El recinto ubicado en el suroeste de RD tiene matriculados 708 estudiantes en tres carreras de licenciatura (Educación Física, Educación Inicial, Educación Primaria Segundo Ciclo).

Para la realización de esta investigación se utilizó una metodología mixta de investigación, mediante la aplicación de una encuesta electrónica a 120 estudiantes de siete secciones del recinto, representando $16,1 \%$ de la población estudiantil. El instrumento contuvo doce preguntas cerradas y una abierta acerca de la accesibilidad tecnológica y de la implementación de clases en línea debido a la emergencia educativa (https://forms.gle/j27z5kdW6baZhbQR6).

En el enfoque cuantitativo, se realizó un análisis estadístico descriptivo e inferencial en el programa $\mathrm{R}$ en el cual se realizó la prueba t pareada (t.test en R) para comparar las medias entre dos grupos de muestras relacionadas con un intervalo de confianza de $95 \%$. Por otra parte, se solicitaron datos a la dirección académica de la institución acerca de las planificaciones implementadas por los docentes, con la finalidad de conocer las herramientas digitales utilizadas, así como la naturaleza de las actividades, evaluativas y no evaluativas, propuestas por los maestros durante el período de la virtualización de las clases. Dichos datos se analizaron en el programa de Excel.

Las herramientas fueron clasificadas en las siguientes categorías: Comunicación directa (correos electrónicos, WhatsApp, comunicación telefónica por compañía); Plataformas educativas (Classroom, Edmodo, Facebook); De encuentro simultáneos (Google Meet, Jitsi Meet, Zoom, Skype); Trabajo en la nube (Google Drive, Google Documents); Creación, edición y publicación de vídeos (Youtube, Edpuzzle); Creación de espacio en la web (Blogspot, Google Site); Formularios (Formularios Google, Formularios Office 365).

Las actividades propuestas por los docentes durante la clases virtuales, se agruparon considerando las siguientes categorías: Producción escrita (propuestas de investigación, informes, ensayos, análisis, historias de vida, reportes escritos, estudios de casos, artículos, diarios reflexivos, bitácoras, resúmenes); Diagramación digital (diapositivas, cuadros comparativos, mapas conceptuales, diseño de esquemas, brochures, infogramas, fotogramas, diseño de site o blog, líneas de tiempo, boletines informativos); Expositivas (exposiciones, debates); Creación y edición de videos; Guías de actividades (guías de preguntas, guías prácticas, actividades 
guiadas); De naturaleza didáctica (Planificaciones, diseño de instrumentos, diseños de recursos, diseño de guías didácticas); Participación de foros; Formularios; Proyectos; Carpetas de evidencias.

En el enfoque cualitativo, el análisis descriptivo fue realizado en el programa Atlas.ti a partir de la importación de las respuestas de la pregunta abierta del cuestionario. Después de esto, las respuestas de los estudiantes fueron leídas y codificadas. La codificación fue realizada inicialmente de forma no estructurada y después de forma jerárquica, relacionando códigos específicos a otros más generalizados. A partir de la vinculación de códigos fueron construidas redes semánticas con los códigos creados. En estas, se consideró la frecuencia de los enraizamientos (E), que corresponde al número de citas en que un código aparece y la densidad de los códigos (D), que representa el número de conexiones con otros códigos en las redes semánticas.

Mapas con la localización de las viviendas de los estudiantes fueron elaborados utilizando el programa QGis. Para esto, la base de datos GeoNames fue utilizada para la obtención de coordenadas geográficas de las ciudades y sectores dónde viven los estudiantes entrevistados. En el mapa, los indicadores del Índice de Desarrollo Humano (IDH) fueron obtenidos en la plataforma del Programa de las Naciones Unidas para el Desarrollo (Programa de las Naciones Unidas para el Desarrollo [PNUD-RD], 2019).

\section{Resultados}

\subsection{Accesibilidad tecnológica}

De los 120 estudiantes que recibieron la encuesta, 101 respondieron, representando 14,3\% de la población del recinto. Los datos cuantitativos muestran que 58 estudiantes (57,4\%) de los 101 encuestados vivían en la residencia universitaria al momento de la interrupción de las clases presenciales. Esta residencia ofrecía tres comidas diarias, además de dos meriendas, acceso a biblioteca, computadoras e internet. De estos, 38 viven ahora en provincias con nivel de IDH Medio Bajo (23 estudiantes de San Juan, 13 de Azua y 2 de Barahona) y 20 en provincias con IDH Bajo (10 de Elías Piña, 6 de Bahoruco, 3 de Independencia y 1 de Pedernales) (Figura 1).

\section{Figura 1}

Mapa relacionando el IDH de República Dominicana con las localidades donde viven los estudiantes encuestados

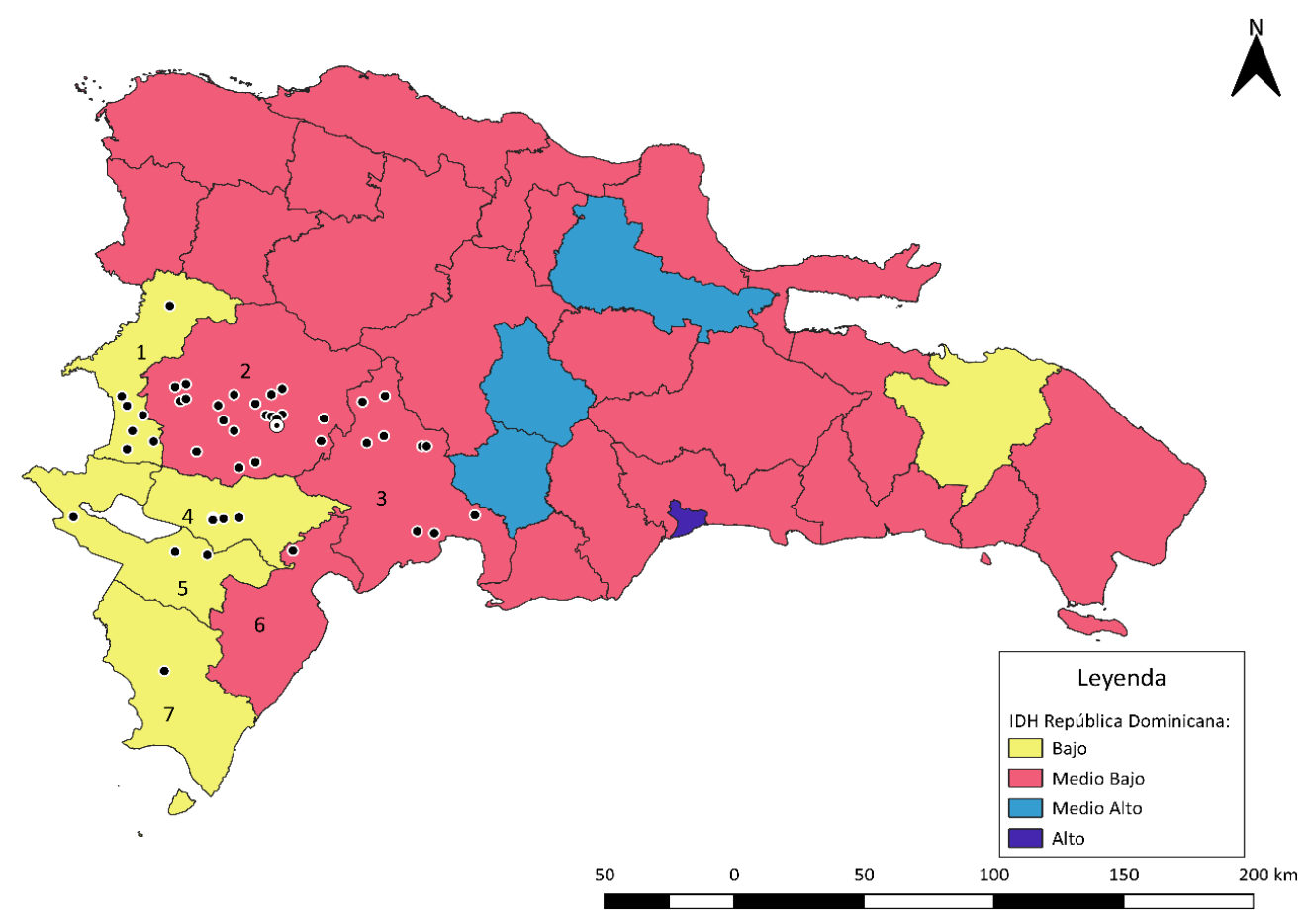

Nota. Estudiantes encuestados (puntos negros), sede universitaria (punto blanco, con negro al centro). Provincias: 1. Elías Piña, 2. San Juan, 3. Azua, 4. Bahoruco, 5. Independencia, 6. Barahona, 7. Pedernales. 
Con relación a los equipos tecnológicos utilizados por los estudiantes durante las clases a distancia, 58 estudiantes $(57,4 \%)$ utilizaron computadoras y teléfonos, 30 estudiantes $(29,7 \%)$ utilizaron solo teléfono, 12 solo computadora (11,9\%) y un estudiante uso tableta y teléfono (1\%). Cuando comparamos la accesibilidad a equipos tecnológicos entre estudiantes que viven en la residencia universitaria y que ahora están en sus hogares (ER) con respecto a aquellos que viven en sus hogares (ENR), no encontramos diferencias significativas con relación al uso de equipos tecnológicos (Cuadro 1).

Cuadro 1

Accesibilidad a equipos tecnológicos en estudiantes residentes (ER) y no residentes en el recinto universitario (ENR)

\begin{tabular}{lrrrrrrr}
\hline & & ER & & ENR & & $T$ & $p$ \\
\cline { 2 - 7 } & & $\mathbf{n}$ & $\%$ & $\mathbf{N}$ & $\%$ & \\
\hline Computadora y teléfono & 33 & 56,9 & 25 & 58,1 & 1,05 & 0,30 \\
Teléfono & 19 & 32,8 & 11 & 25,6 & 1,47 & 0,15 \\
Computadora & 6 & 10,3 & 6 & 14 & 0 & 1 \\
Tabletay teléfono & - & - & 1 & 23 & - & - \\
\hline
\end{tabular}

Nota. Intervalo de confianza de 95\%. ${ }^{*}$ Diferencias significativas para valores de $\mathrm{p}<0,05 \mathrm{~N}=101$.

Con relación a la accesibilidad a internet, 47 estudiantes (46,5\%) contrataron planos personales de datos móviles para las clases virtuales, $27(26,8 \%)$ poseen internet banda larga en la casa, 13 (12,9\%) comparten planos de datos móviles con familiares o vecinos, 8 (7,9\%) utilizan tanto la red-wifi como paquetes de datos personales y 6 (5,9\%) utilizan internet compartida de algún familiar o vecino. La relación de accesibilidad a internet, entre ER y ENR, demuestra una diferencia significativa del uso de paquetes de datos móviles entre ellos $(t=2,55 ; p=0,012)$, así como del uso de internet banda larga $(t=2,57 ; p=0,012)$ (Cuadro 2).

Cuadro 2

Accesibilidad a internet en estudiantes residentes (ER) y no residentes en recinto universitario (ENR)

\begin{tabular}{|c|c|c|c|c|c|c|}
\hline & \multicolumn{2}{|c|}{ ER } & \multicolumn{2}{|c|}{ ENR } & \multirow{2}{*}{$T$} & \multirow{2}{*}{$p$} \\
\hline & $\mathbf{N}$ & $\%$ & $\mathbf{N}$ & $\%$ & & \\
\hline Datos móviles personales & 32 & 55,2 & 15 & 34,9 & 2,55 & $0,012^{*}$ \\
\hline Datos móviles personales compartidos & 8 & 13,8 & 5 & 11,6 & 0,83 & 0,408 \\
\hline Red-wifi compartida & 6 & 10,3 & - & - & - & - \\
\hline Red-wifi de la casa & 7 & 12,1 & 20 & 46,5 & 2,57 & $0,012^{*}$ \\
\hline Red-wifi de la casa y datos móviles personales & 5 & 8,6 & 3 & 7 & 0,71 & 0,482 \\
\hline
\end{tabular}

Nota. Intervalo de confianza de $95 \% .{ }^{*}$ Diferencias significativas para valores de $\mathrm{p}<0,05 . \mathrm{N}=101$.

Con relación a la preparación tecnológica, 59 estudiantes $(58,4 \%)$ consideran que tienen el conocimiento básico para el uso de computadoras, mientras que 38 (37,6\%) consideran que están muy preparados y 4 (4\%) no manejan bien la computadora y sus programas. Referente a la preparación para el uso de teléfonos y tabletas, 67 estudiantes (66,3\%) consideran que están muy entrenados, mientras 32 (31,7\%) poseen conocimientos básicos y 2 (2\%) no manejan bien estos equipos y sus funciones.

\subsection{Preparación docente para la enseñanza virtual}

Con relación a los docentes, se obtuvo la matriz de planificación de clases virtuales de 44 de estos, lo que representa el 72\% del total del personal académico activo en la institución universitaria. Luego del aná- 
lisis, se organizó la información sobre las herramientas digitales usadas (Cuadro 3) y el tipo de actividad que los docentes asignaron a sus estudiantes (Cuadro 4).

Cuadro 3

Herramientas digitales utilizadas por docentes

\begin{tabular}{lcc}
\hline & \multicolumn{2}{c}{$\begin{array}{c}\text { Número de } \\
\text { docentes }\end{array}$} \\
\cline { 2 - 4 } & $\mathbf{N}$ & $\%$ \\
\hline Comunicación directa & 31 & 70,5 \\
Plataformas educativas & 24 & 54,4 \\
De encuentros simultáneos & 14 & 31,8 \\
Trabajo en la nube & 8 & 18,2 \\
Creación, edición y publicación de vídeos & 4 & 9,1 \\
Creación de espacios en la web & 3 & 6,8 \\
Formularios & 3 & 6,8 \\
\hline
\end{tabular}

Nota. Elaboración propia.

Los resultados muestran que el 70,5\% de los docentes, decidió optar por herramientas que les permitiera tener comunicación directa con sus estudiantes y el 31,8\% utilizó plataformas digitales de encuentros simultáneos, afectando a los estudiantes que presentan problemas de conectividad.

Figura 2

Herramientas utilizadas por los docentes durante el periodo de clases virtuales

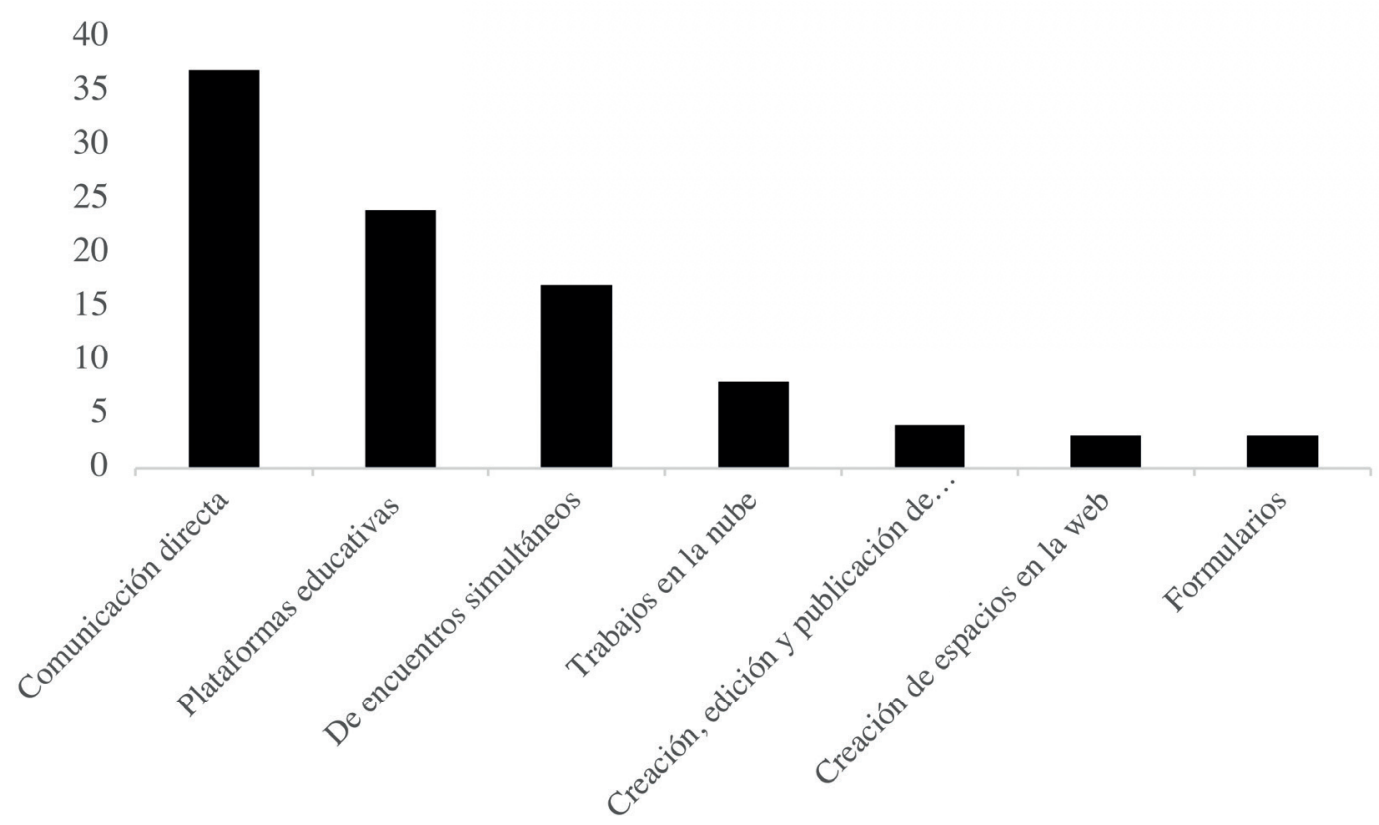

Nota. Elaboración propia.

Por otra parte, 24 de los 44 docentes, utilizaron alguna plataforma educativa para el desarrollo de sus clases virtuales. Llama la atención, que uno de los docentes utilizó la red social Facebook, como plataforma educativa. Finalmente, es de destacar que el 9,1\% implementó herramientas de vídeos y el 6,8\% formularios. 
Los resultados acerca de las actividades muestran que la mayoría de estas estuvieron asociadas a producciones escritas $(72,7 \%)$ y a diagramación digital $(47,7 \%)$. Por otra parte, otro aspecto por destacar es que las actividades, asociadas a la educación a distancia como foros $(20,5 \%)$ y formularios $(18,2 \%)$, obtuvieron porcentajes bajos en comparación a otras actividades planteadas (Cuadro 4).

\section{Cuadro 4}

Naturaleza de las actividades asignadas por los docentes

\begin{tabular}{lcc}
\hline & \multicolumn{2}{c}{$\begin{array}{c}\text { Número de } \\
\text { docentes }\end{array}$} \\
\cline { 2 - 3 } & N & $\%$ \\
\hline Producción escrita & 32 & 72,7 \\
Diagramación digital & 21 & 47,7 \\
Expositivas & 21 & 47,7 \\
Creación y edición de videos & 20 & 45,5 \\
Guía de actividades & 17 & 38,6 \\
De naturaleza didáctica & 16 & 36,4 \\
Participación en foros & 9 & 20,5 \\
Formularios & 8 & 18,2 \\
Proyectos & 7 & 15,9 \\
Carpetas de evidencias & 3 & 6,8 \\
\hline
\end{tabular}

Nota. Elaboración propia.

Con relación a las actividades realizadas por los estudiantes, el promedio fue de 6,7 actividades por cada docente y considerando la carga académica de cada estudiante durante el cuatrimestre, implicaría de 67 a 74 actividades desarrolladas durante las 4 semanas de clases virtuales (Figura 3).

\section{Figura 3}

Naturaleza de las actividades asignadas por los docentes durante el periodo de clases virtuales

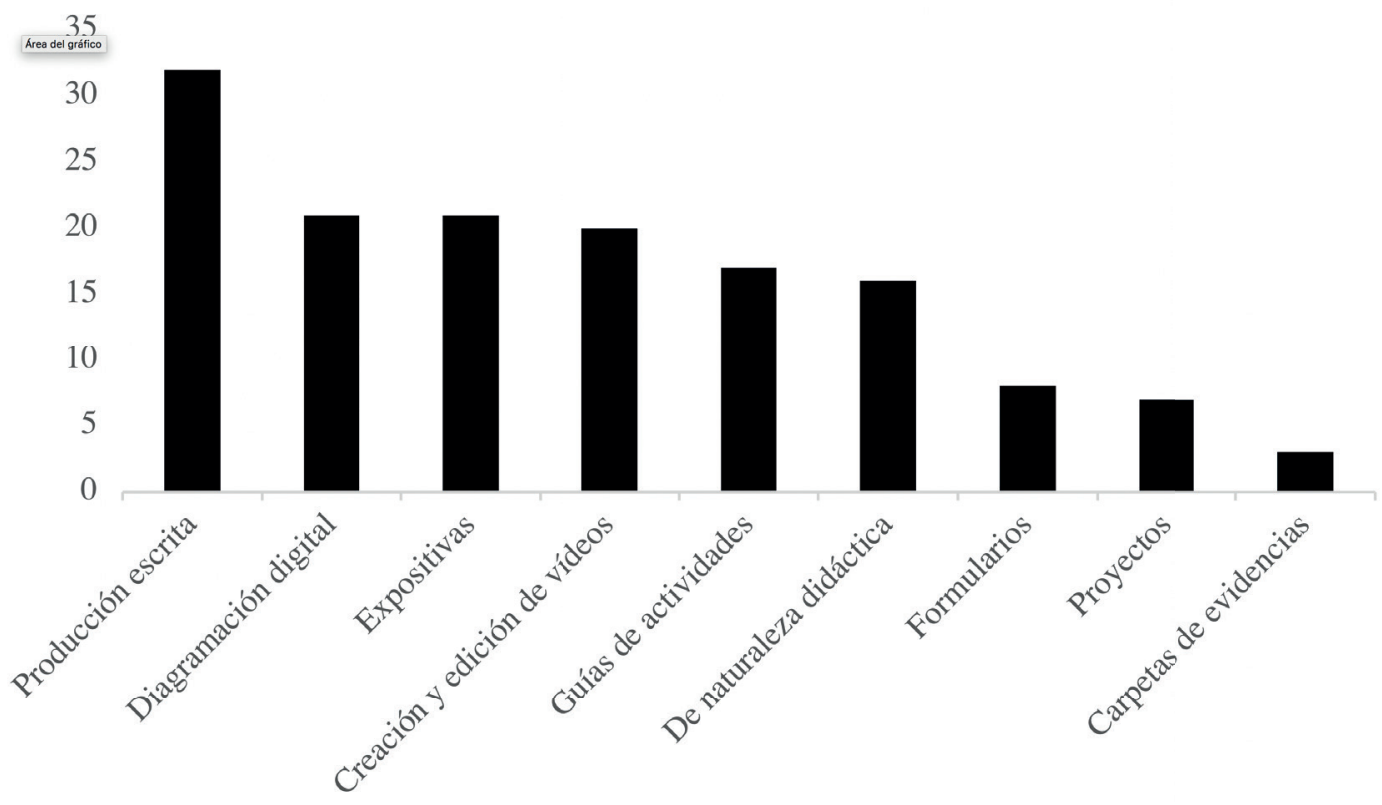

Nota. Elaboración propia. 


\subsection{Percepción estudiantil}

El análisis de las percepciones de los estudiantes sobre la incorporación de la tecnología y la educación a distancia, para finalizar el cuatrimestre generó 210 citas, clasificadas en 54 códigos. De estas citas, $141(67,1 \%)$ resaltaban aspectos negativos y $69(32,9 \%)$ aspectos positivos referentes al proceso. Las percepciones de los estudiantes están asociadas a seis códigos que se refieren a los aspectos positivos y negativos de la implementación de las clases virtuales (Figura 4). Los códigos son referentes a la tecnología, a la parte administrativa de la universidad, a la pandemia por Covid-19, a los docentes y a los estudiantes.

Los aspectos negativos del uso de la tecnología están relacionados a la falta de recursos tecnológicos, 32 enraizamientos $(\mathrm{E})$, que son la falta de computadores y baja velocidad de conexión a internet (Figura 5).

Figura 4

Impresiones principales de los estudiantes acerca de la implementación de clases a distancia, debido a la emergencia remota educativa

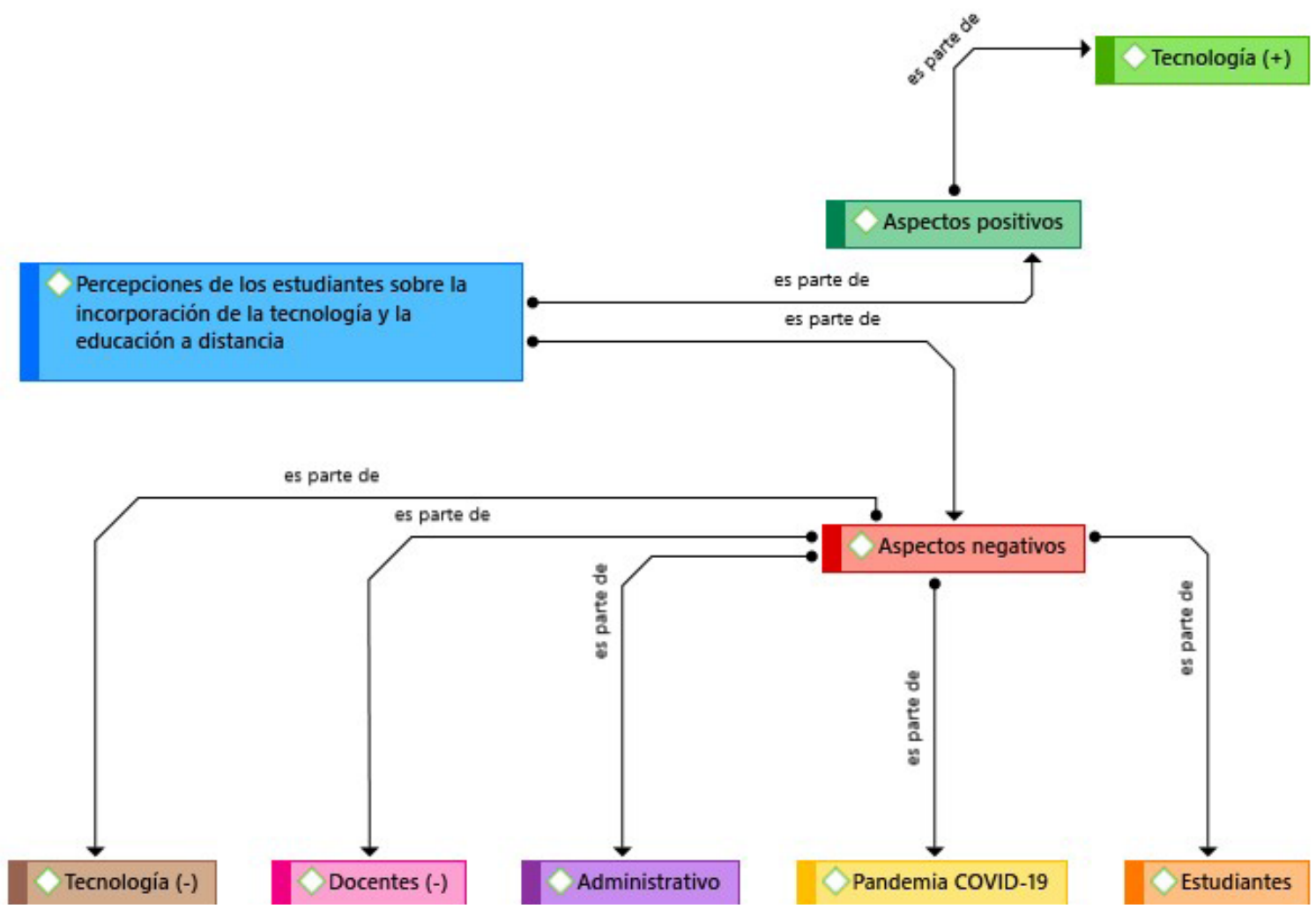

Nota. Elaboración propia.

Según los estudiantes la inaccesibilidad generó incomodidad y estrés por hacer actividades en teléfonos. Las dificultades de utilizar aparatos tecnológicos fueron apuntadas por algunos de los estudiantes del primer año (6 E). Las citas por seguir ejemplifican esto:

Me siento un poco sofocado porque la señal es mala y el internet no funciona bien y mal porque la PC que uso es de dos por lo que me atraso en las tareas. (Estudiante de Educación Primaria, Segundo Ciclo, hombre, $5^{\circ}$ cuatrimestre)

[que] los profesores se pongan en la situación de las personas que no tenemos la facilidad de algunos recursos, sin mencionar que todo lo que nos podría ayudar está cerrado. (Estudiante de Educación Física, hombre, $1^{\circ}$ cuatrimestre)

Es un poco incómodo porque no se tiene una de las herramientas básicas para el desempeño requerido. (Estudiante de Educación Inicial, mujer, $2^{\circ}$ cuatrimestre) 
Las críticas con relación a la administración universitaria están relacionadas con la sobrecarga de asignatura (4 E), la falta de coordinación de los maestros (1 E), la falta de acceso a herramientas tecnológicas $(1 \mathrm{E})$, demora con el pago de estipendio a estudiantes de nuevo ingreso (1 $\mathrm{E})$, lo cual les serviría para pagar internet $(1 \mathrm{E})$, ocasionando incomodidad por no tener dinero para realizar la compra de datos móviles y acceder al servicio (3 E) (Figura 6). Las citas a continuación son ejemplos de sus percepciones:

Antes [de] implementar está estrategia tenía [n] que asegurarse de que todos sus estudiantes contaran con las herramientas necesarias para una clase virtual. (Estudiante de Primaria, Segundo Ciclo, mujer, $5^{\circ}$ cuatrimestre)

Sería bueno que los maestros tomen en cuenta [...] la cantidad de asignaturas que tenemos, para que así podamos cumplir con las demás tareas de las otras asignaturas. (Estudiante de Primaria, Segundo Ciclo, mujer, $5^{\circ}$ cuatrimestre)

Maestros deben ponerse de acuerdo, no sé, crear un grupo entre ellos y hablar sobre el tiempo de cada quien. (Estudiante de Primaria, Segundo Ciclo, mujer, $5^{\circ}$ cuatrimestre)

En realidad, no muy bien, porque tengo que poner internet y pues [...] a mí no me están pagando y la cosa está muy mala. (Estudiante de Primaria, Segundo Ciclo, hombre, $1^{\circ}$ cuatrimestre)

\section{Figura 5}

Impresiones de los estudiantes acerca del uso de la tecnología en las clases a distancia, debido a la emergencia remota educativa

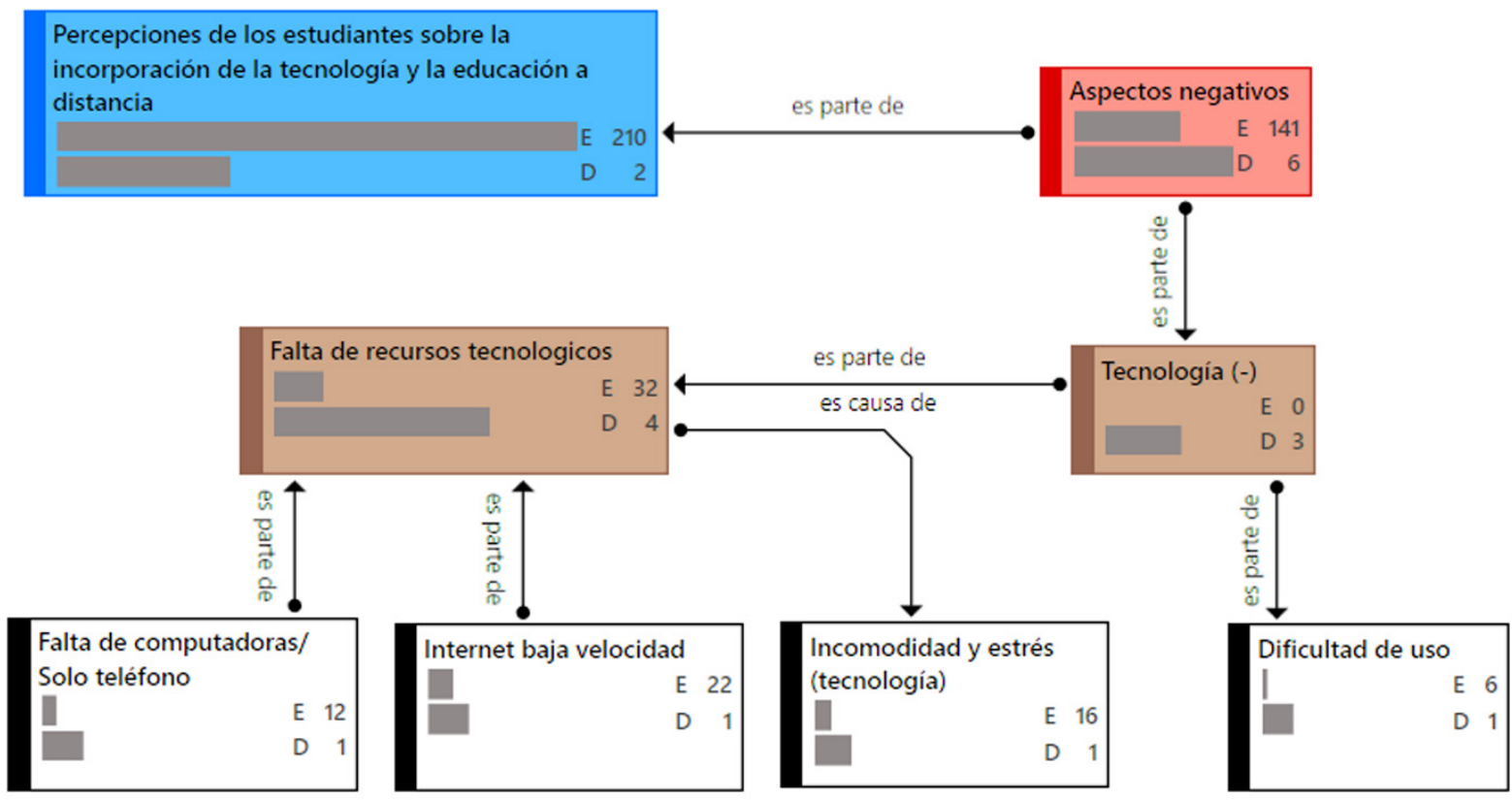

Nota. E: Número de citas vinculadas al código. D: Cantidad de códigos vinculados. 


\section{Figura 6}

Impresiones de los estudiantes acerca del papel de la administración universitaria durante la implementación de clases a distancia debido a la emergencia remota educativa

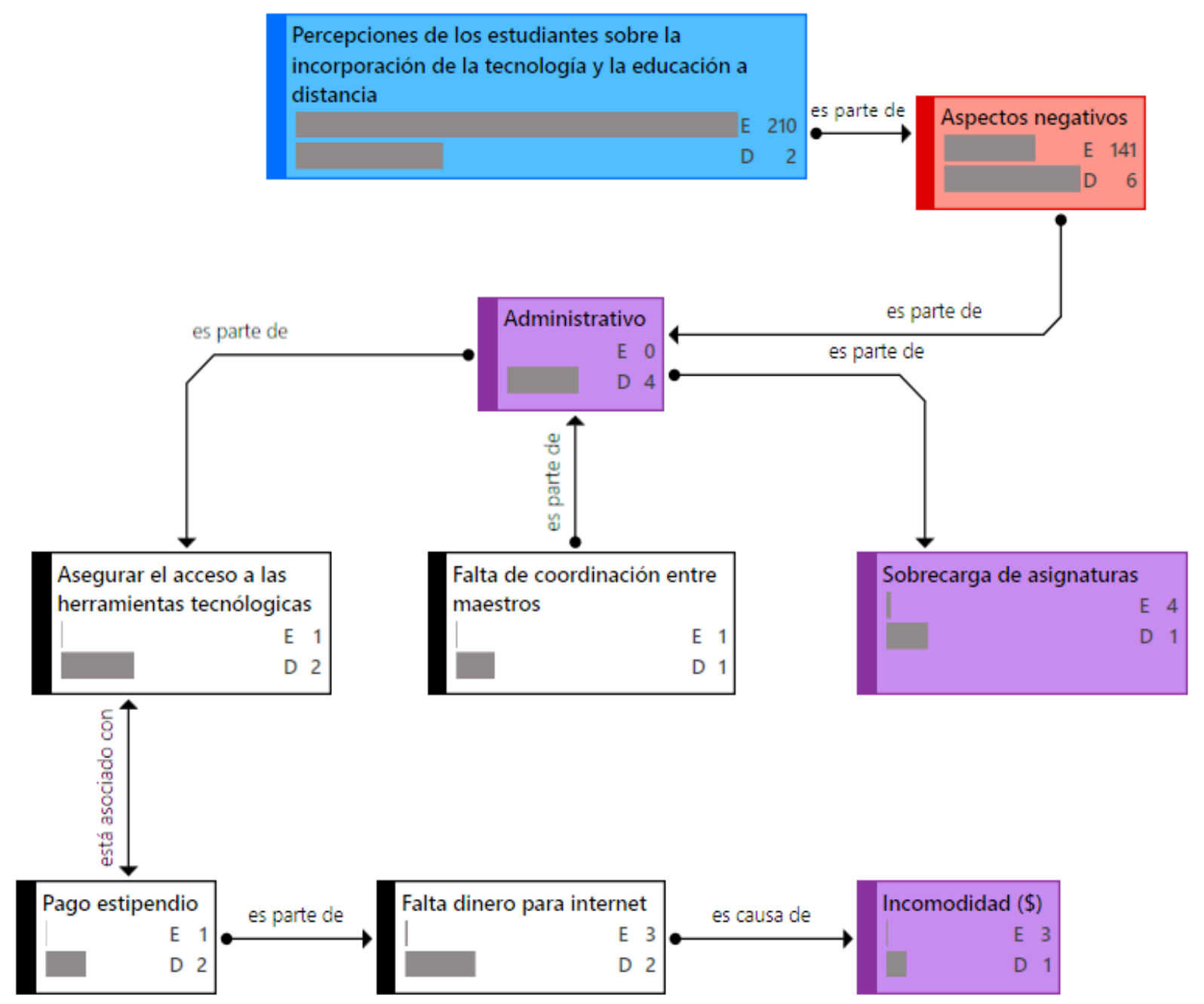

Nota. E: Número de citas vinculadas al código. D: Cantidad de códigos vinculados.

Respecto a las impresiones relacionadas con la pandemia y las acciones tomadas para minimizar el contagio, como el distanciamiento social (3 E), tiene como consecuencias el cierre del comercio, lo que dificulta la realización de recargas $(3 \mathrm{E})$ y la ansiedad debido al aislamiento $(2 \mathrm{E})$. El exceso de información relacionada y el sensacionalismo, genera en los estudiantes falta de concentración $(1 \mathrm{E})$, preocupación personal o por familiares que viven en el exterior (2 E) (Figura 7).

Las citas abajo indican las percepciones de los estudiantes referentes a la pandemia:

Es algo muy turbulento ya que estamos viviendo unos momentos de crisis general, esa causa no nos permite pensar bien ya que todos tenemos familiares que están en esos países más afectados, lo único que quiero [es] que todos salgamos bien de esto. (Estudiante de Educación Física, hombre, $1^{\circ}$ cuatrimestre)

No hay como poner el internet porque no hay recargas ni tarjetas en ningún lado. (Estudiante de Educación Física, hombre, $1^{\circ}$ cuatrimestre)

El estar encerrado no me gusta. [...] [muchos no saben que] estamos en casa sin saber a la ansiedad y la depresión que nos enfrentamos. (Estudiante de Educación Primaria Segundo Ciclo, mujer, $5^{\circ}$ cuatrimestre) 
Figura 7

Efectos causados por la pandemia de la Covid-19 y sus acciones de mitigación en los estudiantes

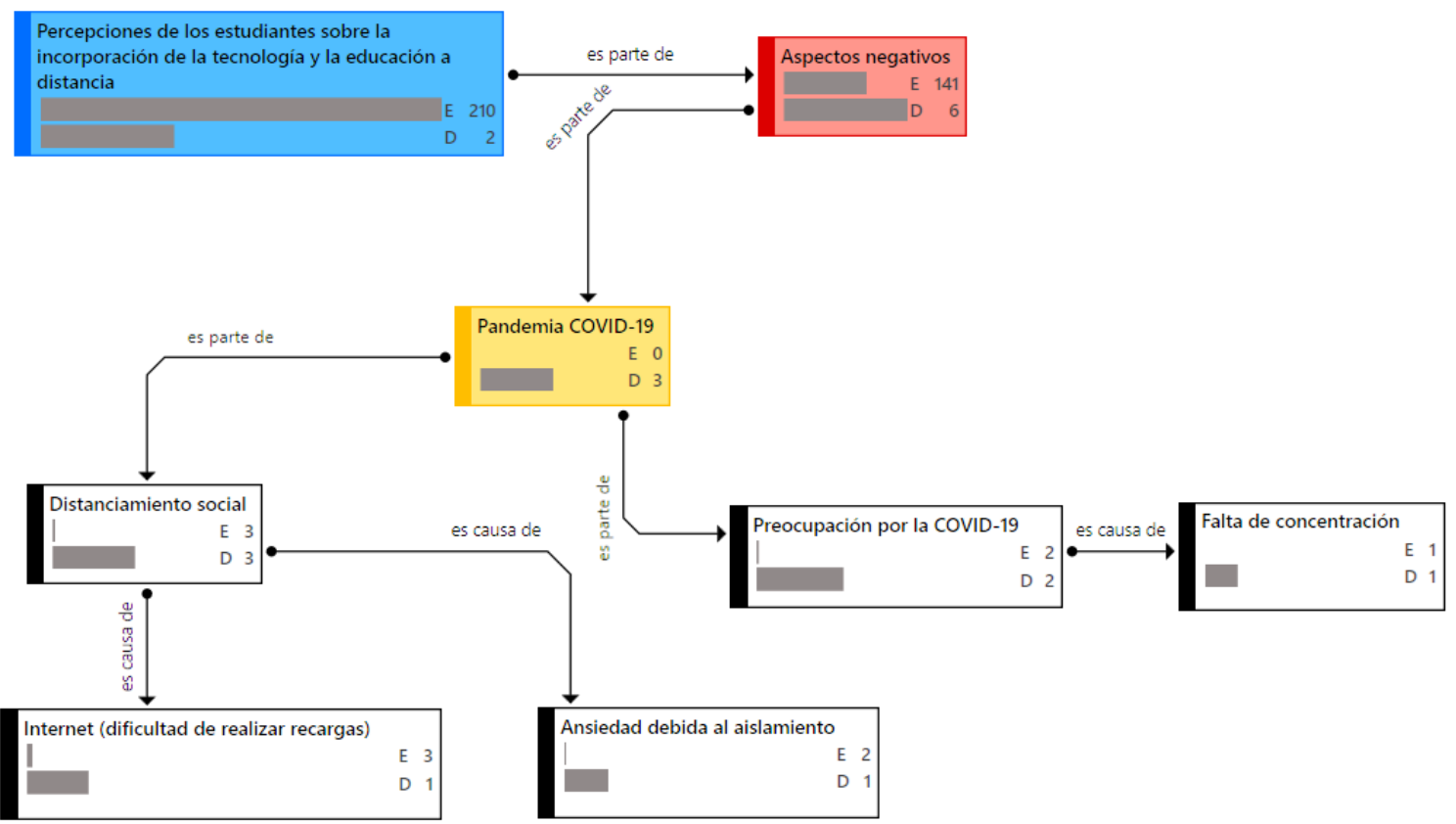

Nota. E: Número de citas vinculadas al código. D: Cantidad de códigos vinculados.

Los estudiantes afirman que los maestros son insensibles, pues falta empatía y comprensión frente a la emergencia educativa, la enseñanza a distancia y la preocupación por la crisis debido a la pandemia por Covid-19 (Figura 8). Como ejemplo, algunos estudiantes mencionan que:

Muchos profesores digo algunos por qué no son todos, abusan de esto que estamos en casa sin saber a la ansiedad y la depresión que nos enfrentamos. (Estudiante de Educación Primaria Segundo Ciclo, mujer, $5^{\circ}$ cuatrimestre)

Este fue un cambio repentino que se debe analizar, tratar de ubicarnosy reflexionar por la situación que estamos viviendo actualmente, es cuestión de tratar de ver un poquito más allá, ser empáticos y entender. (Estudiante de Educación Primaria Segundo Ciclo, mujer, $5^{\circ}$ cuatrimestre)

Entiendo que es una situación que nadie se esperaba, pero deberían tener un poco de consideración con nosotros los estudiantes. (Estudiante de Educación Primaria Segundo Ciclo, mujer, $5^{\circ}$ cuatrimestre)

Un aspecto muy estimado por los estudiantes se refiere a las tareas asignadas por los maestros (Figura 8). De acuerdo con ellos, los maestros generaron sobrecarga de tareas, (42 E) y consecuentemente estrés (25 E), cansancio (14 E) y en algunos casos problemas de salud ( $5 \mathrm{E}$ ). Además, los docentes designaron tareas muy difíciles (5 E) y en horarios y días inapropiados (10 E) y no llevaron en cuenta el tiempo necesario para su realización y (16 E), frustrando los estudiantes que no lograron entregar a tiempo (3 E) (Figura 8). Vemos algunas de las opiniones de los estudiantes sobre estos aspectos:

Algunos docentes te hablan de tareas en horas indebidas. Cómo por ejemplo a las 12 a.m. (Estudiante de Educación Primaria Segundo Ciclo, mujer, $5^{\circ}$ cuatrimestre)

Muy estresada, debido a que todos los profesores dejan muchas tareas y no dan el tiempo suficiente para realizarla. (Estudiante de Educación Primaria Segundo Ciclo, mujer, $4^{\circ}$ cuatrimestre)

Muy saturada algunos profesores aprovechan que estamos en casa y suponen que estamos vagos para aturdirnos con un billón de tareas que en las clases presenciales nunca habríamos hecho, o como es el caso de un maestro al cual le entregamos un trabajo, que hasta calificó y 
ahora quiere que se lo volvamos a hacer, sabiendo que se lo entregamos en físico y que todo eso se quedó en la residencia. (Estudiante de Educación Primaria Segundo Ciclo, mujer, $5^{\circ}$ cuatrimestre)

Por otra parte, lo que está siendo afectada es nuestra salud mental debido a la sobrecarga de tareas que nos están poniendo los maestros, simplemente basándose en el argumento de que "los estudiantes sólo están durmiendo y comiendo en sus casas" cosa que en realidad no es así ya que estamos intentando cumplir al máximo todas las asignaciones. (Estudiante de Educación Primaria Segundo Ciclo, mujer, $4^{\circ}$ cuatrimestre)

Me siento estresada, sufro de los riñones y al durar tanto tiempo sentada me duelen bastante, me siento cansada. (Estudiante de Educación Primaria Segundo Ciclo, mujer, $5^{\circ}$ cuatrimestre)

Otra opinión de los estudiantes, que apunta hacia los maestros es que ellos están preocupados en designar tareas solamente para cumplir con los programas de la asignatura sin considerar el aprendizaje (4 E) (Figura 8, Cuadro 9), como considera este estudiante:

La gran mayoría de los profesores no se están preocupando por el aprendizaje de sus estudiantes sino más bien por dejar asignaciones y ese no es el trabajo de un docente ni debe ser su objetivo. (Estudiante de Educación Primaria Segundo Ciclo, mujer, $5^{\circ}$ cuatrimestre)

Con relación a la adaptación a las clases virtuales, los estudiantes relatan dificultades de aprendizaje (13 E) y preferencia por clases presenciales (6 E) (Figura 9). Los estudiantes señalan también que tienen miedo de no obtener los conocimientos necesarios para su formación (1 E). Además, se sienten incomodos con la implementación de las clases virtuales (12 E), debido a la dificultad de concentración en la casa (2 E) de manejar el tiempo de estudio (1 E), y, además de considerar el hogar inestable (1 E) para estudiar porque tienen que lidiar con las tareas domésticas ( $2 \mathrm{E}$ ). Esto puede conllevar a la deserción universitaria como apuntó una estudiante (Figura 9).

\section{Figura 8}

Impresiones de los estudiantes acerca del desempeño de los maestros durante las clases a distancia

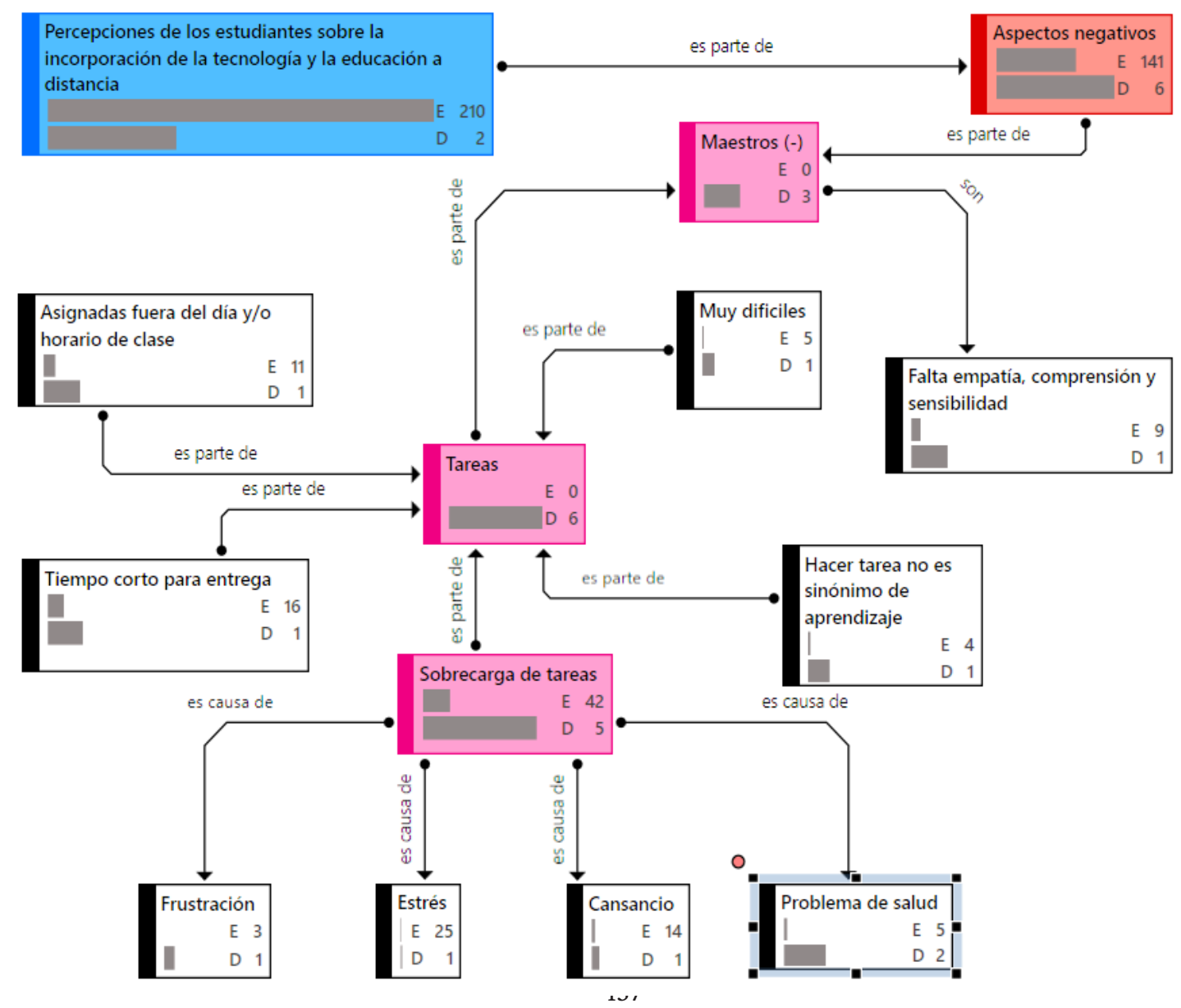


Las siguientes citas expresan las dificultades encontradas por los estudiantes durante el proceso de clases a distancia:

En ocasiones cuando tengo [que] hacer alguna tarea en mi casa me ponen a cosinar [cocinar] o hacer otra cosa y no sé a qué hecharle [echarle] mano. (Estudiante de Educación Primaria Segundo Ciclo, mujer, $5^{\circ}$ cuatrimestre)

Pero el estudiar desde casa en un ambiente donde hay personas en otras ocupaciones es muy incómodo, ya que eso distrae bastante. (Estudiante de Educación Primaria Segundo Ciclo, mujer, $4^{\circ}$ cuatrimestre)

Lo que en verdad me asusta de estas clases es no poder obtener los conocimientos necesarios sobre algún tema, el cual pueda afectarme más adelante en una clase presencial. (Estudiante de Educación Primaria Segundo Ciclo, mujer, $5^{\circ}$ cuatrimestre)

Debido a que tengo que ayudar con los quehaceres y también cumplir con las tareas, a veces me dan ganas de rendirme, pero luego digo que no, que debo seguir hasta el final. (Estudiante de Educación Primaria Segundo Ciclo, mujer, $5^{\circ}$ cuatrimestre)

\section{Figura 9}

Impresiones negativas intrínsecas a los estudiantes acerca de la implementación de clases a distancia

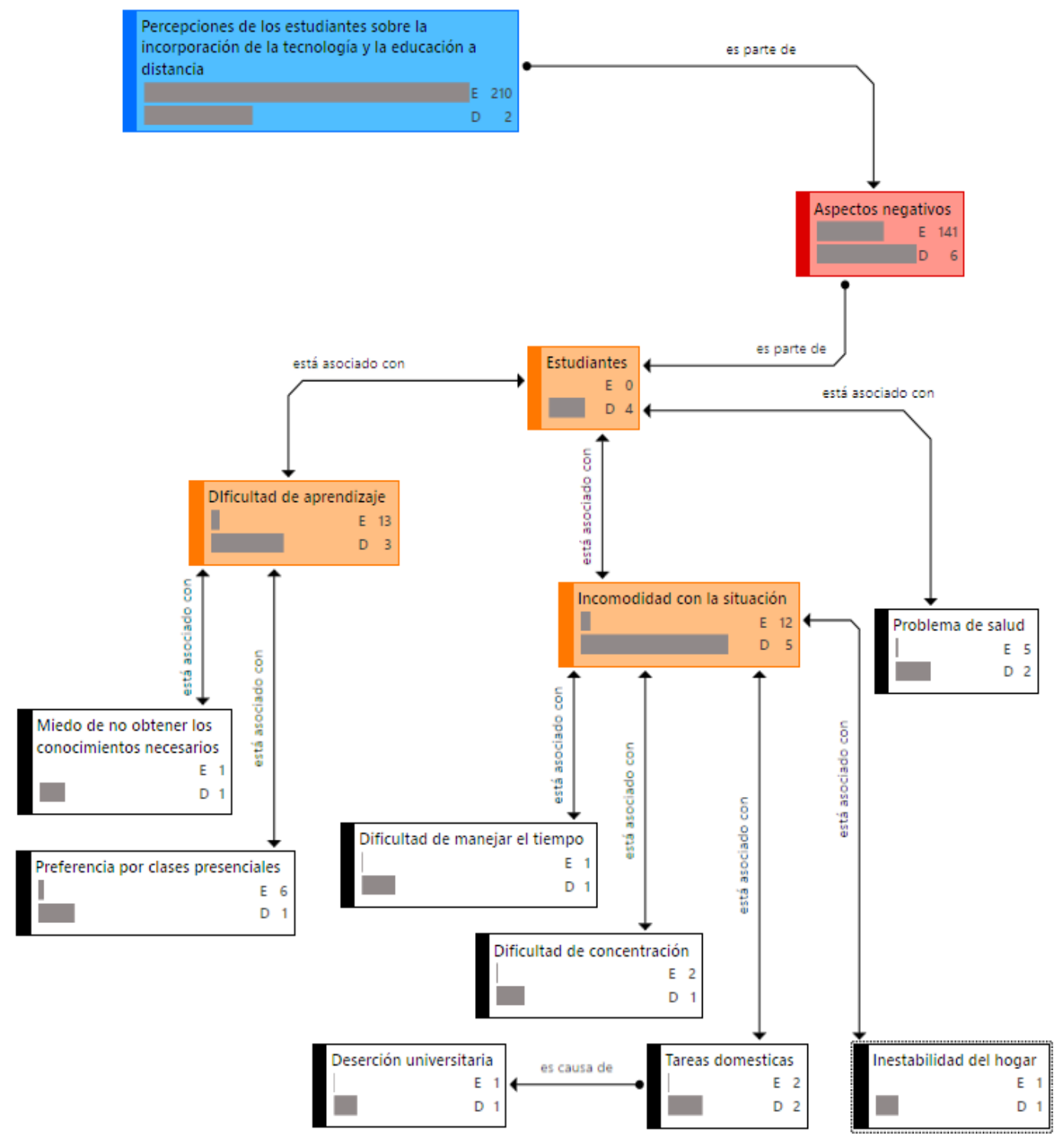

Nota. E: Número de citas vinculadas al código. D: Cantidad de códigos vinculados. 
Las percepciones positivas de los estudiantes con el proceso de virtualización están agrupadas en tres códigos referentes al uso de la tecnología, a la posibilidad de la continuidad de los estudios y a la comodidad en la utilización de la tecnología y de estar en la casa (Figura 10). Los estudiantes se sienten cómodos con la implementación de las clases en línea (43 E), por la comodidad de estar en sus casas y no vivir en la residencia universitaria (1 E) (Figura 10). Un aspecto destacado por los estudiantes fue el poder dar continuidad al cuatrimestre (10 E) permitiendo la culminación mediante sus esfuerzos (1 E). Algunos apuntan que la tecnología facilita el aprendizaje, proporcionando un mejor dominio de los temas $(3 \mathrm{E})$ y que el proceso de enseñanza y aprendizaje mediante el uso de la tecnología es una innovación (8 E). Algunos tienen también una percepción positiva del proceso debido a la facilidad del uso de herramientas tecnológicas ( $3 \mathrm{E}$ ) y por tener una buena conexión de internet (1 E). Las citas abajo resaltan los aspectos positivos de la implementación de las clases virtuales:

Me siento bien ya que es un buen método que han incorporado para que los estudiantes no perdamos el cuatrimestre debido a esta pandemia que nos está afectando. (Estudiante de Educación Física, hombre, $1^{\circ}$ cuatrimestre)

Me siento muy bien porque en situaciones como la que nos encontramos ahora mismo ha sido una herramienta de mucha utilidad, ya que, si no hubiese sido por la tecnología, iba a perder el cuatrimestre. (Estudiante de Educación Inicial, mujer, $1^{\circ}$ cuatrimestre)

Excelente siento mejor dominio de los temas porque soy yo quien los investiga y estudia siento que eso me ha favorecido mucho en el aspecto intelectual. (Estudiante de Educación Primaria, hombre, $5^{\circ}$ cuatrimestre)

Figura 10

Impresiones positivas de los estudiantes acerca de la implementación de clases a distancia debido a la emergencia remota educativa

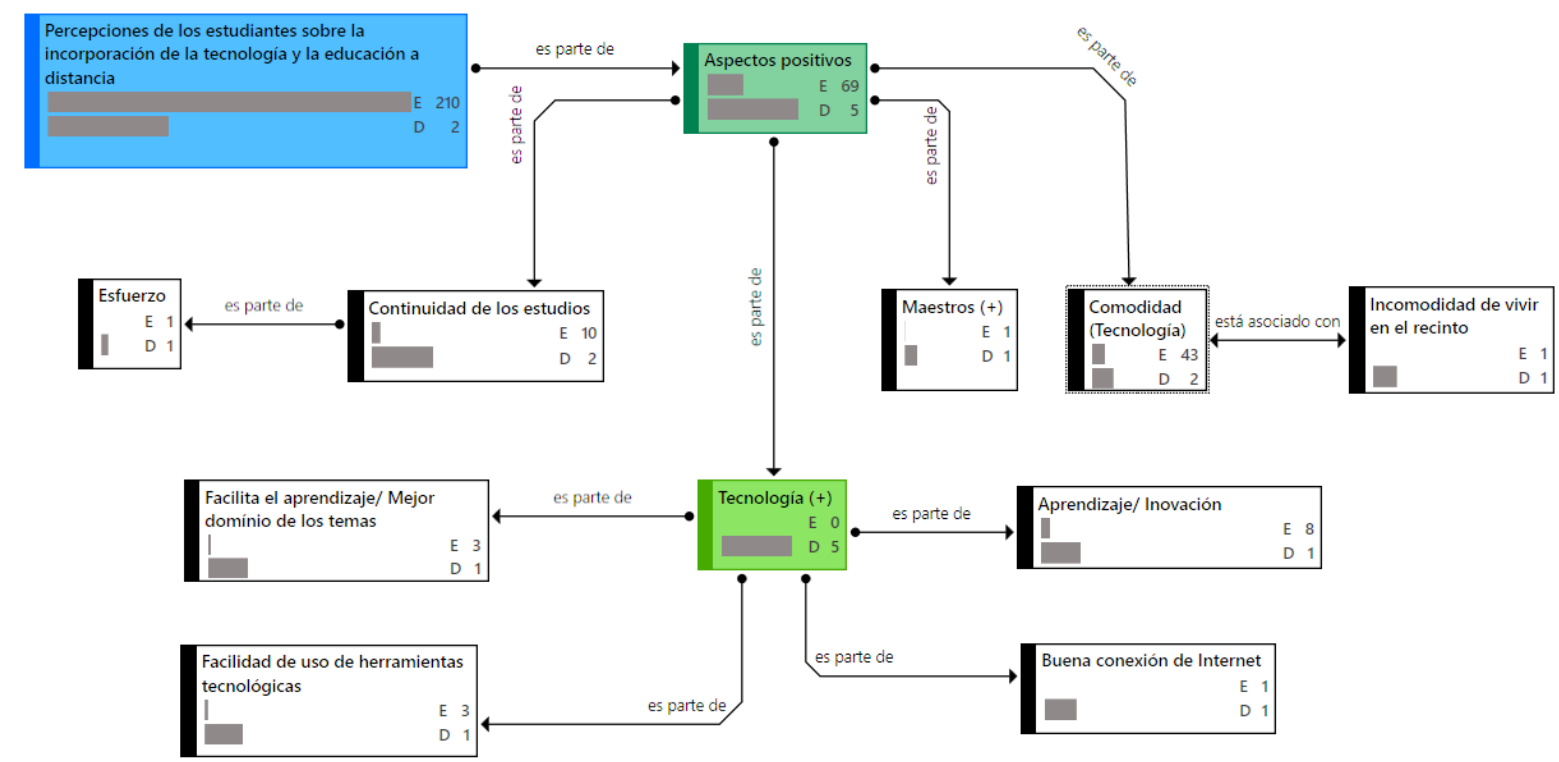

Nota. E: Número de citas vinculadas al código. D: Cantidad de códigos vinculados.

\section{Discusión}

Con el objetivo de continuar los procesos iniciados con los estudiantes y ser un apoyo social tanto para el alumnado como para su familia, los ministerios de educación de diversos países incentivaron a las instituciones educativas a continuar las clases de forma remota.

A pesar de las buenas intenciones, en RD muchas instituciones de educación superior optaron por clases en línea mediante el uso de las TIC sin la debida preparación de las instituciones, profesores y estudiantes 
(UNESCO-IESALC, 2020). Esto incluye la falta de planificación institucional, de accesibilidad tecnológica, y de estrategias y competencias docentes para la implementación de educación en línea.

Los datos de los estudiantes encuestados confirman la falta de accesibilidad al internet en el suroeste de RD (ONE, 2018), aspecto que constituye una barrera para el uso de las TIC y la implementación efectiva de la educación en línea en la región suroeste de RD y en el país (Nye, 2015; Scagnoli, 2009). Además de esto, en áreas rurales o aisladas de las grandes ciudades, como es el caso del suroeste dominicano, hay poca señal y/o baja velocidad de conexión de internet. Como consecuencia, los estudiantes presentan dificultades para acceder a contenidos multimedia, participar de actividades sincrónicas mediante el uso de video e incluso acceder a plataformas educativas.

Además del problema de conectividad, el uso de ordenadores por los estudiantes no es común. Las computadoras son productivas cuando se deben realizar tareas complejas, como la creación de gráficos, análisis de datos y producciones escritas, además, algunos programas de computación no poseen versiones para teléfonos móviles (Nye, 2015). No obstante, la falta de computadoras puede no ser un problema para la educación a distancia, desde que las instituciones y docentes sepan y elaboren actividades convenientes para el aprendizaje a través del uso de teléfonos (Bates, 2005, 2015).

Los resultados indican que los teléfonos son los principales equipos tecnológicos utilizados por los alumnos para el aprendizaje virtual, lo que evidencia la baja accesibilidad de computadoras, confirmando datos de encuestas nacionales y de la literatura académica (Nye, 2015; ONE, 2018). Al mismo tiempo, los resultados indican que las actividades asignadas por los maestros durante la emergencia remota educativa no estaban adaptadas para la realización en teléfonos, evidenciando la falta de planificación, coordinación y organización entre la institución, profesores y estudiantes.

La inaccesibilidad tecnológica además de ser una barrera para la educación a distancia también refuerza las desigualdades sociales (Nye, 2015; Tait y O’Rourke, 2014; Wright, Dhanarajan y Reju, 2009). Al mismo tiempo, en la percepción de los estudiantes la falta de computadoras y de acceso al internet genera incomodidad y frustración por no lograr realizar algunas actividades o terminarlas a tiempo, al tener que hacerlas desde un celular. Esto puede ser evitado mediante una consulta sobre la accesibilidad tecnológica de los estudiantes antes del inicio de las clases en línea (Bates, 2005), para que los docentes conozcan y consideren la realidad de los estudiantes adaptando la enseñanza virtual a las condiciones del alumnado.

La falta de preparación de las instituciones y de los docentes para lidiar con la emergencia remota educativa se evidencia en la predominancia de las percepciones negativas por parte de los estudiantes acerca de la implementación de la educación en línea. Las matrices de planificación recolectadas evidenciaron que, a pesar de que se tenía constante comunicación entre docentes y estudiantes, estas se limitaban a aclarar las instrucciones de las actividades evaluativas que debían ser realizadas. Según Anderson et al (2001), en la educación virtual, es necesario un dialogo constante entre docentes y estudiantes que incluyan lecturas, exploraciones web, ejercicios y proyectos individuales y colaborativos. De igual manera, los roles que desempeñan los docentes en la educación presencial deben ser transformados con el propósito de asegurar el cumplimiento de cada uno de los objetivos.

Con relación a los elementos primordiales para la docencia en línea (Bates, 2015; Ní Shé et al., 2019), en el caso del suroeste de RD, se acentuó el uso de estrategias gerenciales (gestión de actividades) al momento de la incorporación de las clases virtuales. Como consecuencia, las funciones y competencias pedagógicas, afectivas, sociales, tecnológicas, evaluadoras y facilitadoras fueron poco desarrolladas, lo que podría indicar la falta de capacitación de los maestros para la educación en línea y la adaptación de los métodos de clase presencial a virtual sin la debida preparación.

Según Bates (2015), este tipo de clase sin que los elementos y los objetivos de aprendizaje de la educación en línea sean considerados, es caracterizado como "el vino viejo, en una nueva botella". Esto es demostrado con el uso de herramientas no diseñadas para la educación virtual, como redes sociales y aplicaciones de mensajería, que pueden apoyar el proceso, pero no conducirlo.

Para que los estudiantes obtengan los resultados de aprendizaje esperados en la enseñanza en línea, es necesario, por ejemplo, el uso de recursos multimedia e investigación en el Internet (Ally, 2008). Al mismo tiempo es necesario que los maestros propongan actividades que promuevan procesos cognitivos de 
orden superior (Ally, 2008; Dunlap y Lowenthal, 2018). Esto se podría lograr con la capacitación docente y la adaptación de programas curriculares para la educación en línea.

Con relación a los resultados de aprendizaje de los estudiantes durante la emergencia remota educativa, es importante que estos puedan ser evaluados con el objetivo de verificar la eficacia de las medidas implementadas. Esto principalmente, en el caso de estudiantes de Educación quienes serán los docentes del futuro y cuya experiencia en línea les servirá para perfeccionar su quehacer docente.

En este estudio, los estudiantes expresaron falta de empatía y comprensión por parte de los docentes frente a la situación de enseñanza a distancia, lo que demuestra la falta de la función o competencia afectiva (Coppola, Hiltz y Rotter, 2002). Para ellos, los maestros eran insensibles al asignar tareas en horarios o días inapropiados y exigir la participación en actividades sincrónicas, sabiendo de las dificultades de accesibilidad tecnológicas y los constantes apagones eléctricos en $\mathrm{RD}$, generando estrés en los estudiantes.

La empatía y preocupación por la salud mental de los estudiantes durante los momentos de crisis, debe ser uno de los ejes principales que guíen el quehacer docente. Cao y colaboradores (2020), en su publicación sobre el impacto psicológico de la pandemia por Covid-19 en estudiantes universitarios, encontraron que los efectos económicos, los efectos de la vida diaria y el retraso en las actividades académicas, están correlacionados con síntomas de ansiedad de leve a severa ( $21,3 \%$ leves, $2,7 \%$ moderados y $0,9 \%$ severos), Por lo tanto, la estabilidad emocional y el monitoreo de la salud mental de los estudiantes es importante para confrontar el miedo y la ansiedad causada por el coronavirus (Abdel-Fattah, 2020; Cao et al., 2020).

La epidemia trajo consigo temor y riesgo de contraer una enfermedad potencialmente letal, así como también una fuerte presión psicológica para la población, debido a que se ha interrumpido casi todos los aspectos de la vida diaria y en muchos casos ha generado dificultades económicas por las estrictas medidas de aislamiento (Cao et al., 2020; Polizzi Lynn y Perry, 2020). En el caso de los estudiantes universitarios, algunos factores que influencian el aumento de la ansiedad son el retraso de las clases, vivir en áreas rurales, vivir en familias sin ingresos estables, además de tener familiares o conocidos enfermos por Covid-19 (Cao et al., 2020).

Las condiciones económicas y de vivienda de los estudiantes en el sur de la República Dominicana, pueden llegar a ser un factor de incidencia en la ansiedad e inestabilidad emocional durante la implementación de la educación a distancia. El desbalance entre los recursos económicos, culturales y educativos entre las áreas urbanas y rurales, así como las condiciones sanitarias disminuye las probabilidades de sobrevivir al virus (Cao et al., 2020), hecho que puede afectar la estabilidad emocional de los estudiantes, al percibir la realidad de su vulnerabilidad.

La mayoría de las percepciones negativas de los estudiantes acerca de las funciones de los docentes están relacionadas con las tareas, la sobrecarga académica, el tiempo reducido para realizar las actividades y su complejidad. Estos aspectos puedan estar asociados con las herramientas tecnológicas utilizadas, los tipos de actividades y la falta de instrucciones claras. Esto indica la necesidad de capacitación docente, teniendo en cuenta las diferentes funciones y los elementos primordiales para la docencia en línea (Bates, 2015; Burns, 2011, Dunlap y Lowenthal, 2018; Muñoz, González y Hernández, 2013; Ní Shé et al., 2019; Oyarzun, Martin y Moore, 2020).

Con relación a los aspectos intrínsecos del aprendizaje en línea, los resultados indican que los estudiantes deben ser instruidos a desarrollar competencias como la autorregulación, gestión del tiempo y aprendizaje autónomo, lo que corrobora publicaciones anteriores sobre el tema (Ally, 2008; Berridi y Martínez Guerrero, 2017; Burns, 2011; Martin y Bolliger, 2018), Al mismo tiempo, para superar las dificultades de aprendizaje, es necesario que los docentes utilicen diversos recursos de aprendizaje considerando que los estudiantes aprenden de diferentes formas (Bates, 2015).

Entre los aspectos positivos mencionados por los estudiantes están el uso de la tecnología para continuar los estudios, gestionar el aprendizaje y presentar un mejor dominio de los temas. Esto indica que parte de los alumnos se adaptó al proceso de enseñanza y aprendizaje centrado en el estudiante, que es una característica de la educación a distancia (Burns, 2011; Ramas Arauz, 2015).

La accesibilidad tecnológica, la capacitación y formación de profesores son desafíos para la enseñanza en línea por parte de las instituciones educativas y los docentes. No obstante, acompañar los estudiantes 
en el proceso, conocer sus aspectos socioeconómicos y sus dificultades de aprendizaje son de extrema importancia, principalmente donde los indicadores de calidad educativa y socioeconómicos son proble-máticos, como es el caso de RD y el suroeste del país (OECD, 2019 PNUD-RD, 2013).

Considerando la baja accesibilidad de los estudiantes a la tecnología, son necesarias políticas públicas para el fortalecimiento de infraestructura, en particular en materia de ancho de banda y accesibilidad en el interior del país, además del acceso a equipos tecnológicos. Cabe resaltar que, durante la emergencia educativa, al final del período académico enero-mayo 2020, en la institución universitaria en la cual se hizo esta investigación, se realizó el levantamiento de datos relacionados a la falta de herramientas tecnológicas entre los estudiantes y a partir de esta relación fue posible gestionar la adquisición de computadores por parte del Estado para los estudiantes necesitados, con el fin de lograr continuar estudiando de manera virtual en el periodo académico siguiente (Hoy Digital, 2020).

Una constante evaluación de la implementación de la educación en línea en virtud de la pandemia por Covid-19 es necesaria para que nuevas medidas puedan ser implementadas para corregir los aspectos a mejorar. Al mismo tiempo, se evidencia que el trabajo coordinado de docentes, instituciones educativas y gobiernos es la mejor estrategia para disminuir las brechas sociales relacionadas con la accesibilidad tecnológica y la capacitación profesional. La motivación tanto de docentes como de estudiantes es fundamental para el éxito del proceso pedagógico.

\section{Conclusión}

La educación a distancia ha permitido la inclusión de nuevos estudiantes en nivel terciario en muchas partes del mundo, aumentando la accesibilidad a los cursos universitarios y ofreciendo nuevas posibilidades de capacitación, y consecuentemente empleo y beneficios socioeconómicos mayores. Sin embargo, en la emergencia remota educativa causada por la pandemia de la Covid-19, la adopción rápida de la educación en línea viene generando insatisfacción y exclusión de los estudiantes en virtud de la falta de accesibilidad tecnológica y planificación institucional y en general, falta de preparación de los docentes para esta modalidad educativa. En contradicción, parte de los estudiantes aprueban la implementación del aprendizaje en línea debido a la continuidad de los estudios durante la emergencia educativa y la posibilidad del uso de la tecnología para gestionar el aprendizaje. Es necesario que después de la pandemia, los resultados de aprendizaje puedan ser verificados con el objetivo de evaluar la eficacia de la implementación de la educación sin la debida organización previa. A partir de la experiencia de la emergencia educativa debido a la Covid-19, los ministerios y las instituciones de educación deben establecer planes estratégicos para futuras crisis sanitarias o ambientales.

\section{Agradecimientos}

A todos los estudiantes que contestaron la encuesta en un momento de sobrecarga de tareas y percibieron la importancia de esta investigación. A la Dirección Académica de la institución universitaria donde se desarrolló la investigación por compartir datos acerca de las herramientas tecnológicas y actividades realizadas por los docentes durante la emergencia educativa. LS y DG agradecen a las siguientes instituciones públicas del estado dominicano por la contratación en el programa de Profesores Invitados de Alta Calificación (PIAC) en el acuerdo de cooperación institucional suscrito por el Ministerio de Educación Superior Ciencia y Tecnología (MESCYT), el Ministerio de Educación (MINERD), el Instituto Nacional de Formación y Capacitación del Magisterio (INAFOCAM) y el Instituto de Formación Docente Salomé Ureña (ISFODOSU).

\section{Referencias}

Abdel-Fattah, H. (2020). Emotional intelligence and emotional stability in crises. Journal of Psychiatry and Psychiatric Disorders, 4, 56-62. https://doi.org/10.26502/jppd.2572-519X0090 
Ally, M. (2008). Foundations of educational theory for online learning. En T. Anderson (Ed.), The theory and practice of online learning (pp. 15-44). AU Press.

Álvarez, M., Gardyn, N., Iardelevsky, A. y Rebello, G. (2020). Segregación educativa en tiempos de pandemia: Balance de las acciones iniciales durante el aislamiento social por el Covid-19 en Argentina. Revista Internacional de Educación para la Justicia Social, 9(3), 25-43. https://doi.org/10.15366/riejs2020.9.3.002

Anderson, T., Rourke, L., Garrison, R. y Archer, W. (2001). Assessing teaching presence in a computer conference context. Journal of Asynchronous Learning Networks, 5(2), 1-17. https://doi.org/10.24059/olj.v5i2.1875

Bates, A. W. (2005). Technology, e-learning and distance education. Routledge. https://doi.org/10.4324/9780203463772

Bates, A. W. (2015). Teaching in a digital age. Tony Bates Associates Ltd.

Berridi, R. y Martínez Guerrero, J. I. (2017). Estrategias de autorregulación en contextos virtuales de aprendizaje. Perfiles Educativos, 39(156), 89-102. https://doi.org/10.22201/iisue.24486167e.2017.156.58285

Bingimlas, K. A. (2009). Barriers to the successful integration of ICT in teaching and learning environments: A review of the literature. Eurasia Journal of Mathematics, Science \& Technology Education 5(3), 235-245. https://doi.org/10.12973/ejmste/75275

Burns, M. (2011). Distance education for teacher: Modes, models, and methods. Education Development Center.

Cao, W., Fang, Z., Hou, G., Han, M., Xu, X., Dong, J. y Zheng, J. (2020). The psychological impact of the Covid-19 epidemic on college students in China. Psychiatry Research,287,112934. https://doi.org/10.1016/j.psychres.2020.112934

Cerezo, R., Esteban, M., Sánchez-Santillán, M. y Núñez, J. C. (2017). Procrastinating behavior in computer-based learning environments to predict performance: A case study in moodle. Frontiers in Psychology, 8, 1403. https://doi.org/10.3389/fpsyg.2017.01403

Coppola, N. W., Hiltz, S. R. y Rotter, N. G. (2002). Becoming a virtual professor: Pedagogical roles and asynchronous learning networks. Journal of Management Information Systems, 18(4), 169-189. https://doi.org/10.1080/07421222.2002.11045703

Dunlap, J. C. y Lowenthal, p. R. (2018). Online educators' recommendations for teaching online: Crowdsourcing in action. Open Praxis, 10(1), 79-89. https://doi.org/10.5944/openpraxis.10.1.721

García, C. M., Burgos, D. R., Murillo Estepa, p. y Jaspez Nero, J. F. (2019). Aprender con tecnologías para enseñar con tecnologías en República Dominicana. El programa república digital educación. Revista Iberoamericana de Educación, 79(1), 115-134. https://doi.org/10.35362/rie7913322

Holguín, E. C. y Sandoval, p. (2020). Resistir la Covid-19. Intersecciones en la educación de Ciudad Juárez, México. Revista Internacional de Educación para la Justicia Social, 9(3), 7-23. https://doi.org/10.15366/riejs2020.9.3.001

Hoy Digital. (3 de junio de 2020). MINERD reconoce estudiantes de pedagogía de ISFODOSU. Hoy Digital. https://hoy.com.do/minerd-reconoce-estudiantes-de-pedagogia-de-isfodosu/

Martin, F. y Bolliger, D. U. (2018). Engagement matters: Student perceptions on the importance of engagement strategies in the online learning environment. Online Learning, 22(1), 205-222. https://doi.org/10.24059/olj.v22i1.1092

Muñoz, p. C., González, M. y Hernández, N. (2013). Pedagogical roles and competencies of university teachers practicing in the e-learning environment. The International Review of Research in Open and Distributed Learning, 14(3), 462-487. https://doi.org/10.19173/irrodl.v14i3.1477

Ní Shé, C., Farrell, O., Brunton, J., Costello, E. y Donlon, E. (2019). Teaching online is different: Critical perspectives from the literature. Zenodo. https://doi.org/10.5281/zenodo.3479402

Nye, B. D. (2015). Intelligent tutoring systems by and for the developing world: A review of trends and approaches for educational technology in a global context. International Journal of Artificial Intelligence in Education, 25(2), 177-203. https://doi.org/10.1007/s40593-014-0028-6

Oficina Nacional de Estadística-ONE. (2018). Encuesta nacional de hogares de propósitos múltiples (ENHOGAR-2017). Oficina Nacional de Estadística. 
Organisation for Economic Co-operation and Development - OECD. (2019). Country note, programme for international student assessment (PISA) results from PISA 2018, Dominican Republic. OECD.

Oyarzun, B., Martin, F. y Moore, R. L. (2020). Time management matters: Online faculty perceptions of helpfulness of time management strategies. Distance Education, 41(1), 106-127. https://doi.org/10.1080/01587919.2020.1724773

Polizzi, C., Lynn, S. J. y Perry, A. (2020). Stress and coping in the time of Covid-19. Clinical Neuropsychiatry, 17(2), 59-62. https://doi.org/10.36131/CN20200204

Programa de las Naciones Unidas para el Desarrollo - PNUD. (2019). Informe sobre desarrollo humano 2019. PNUD.

Programa de las Naciones Unidas para el Desarrollo, Republica Dominicana - PNUD-RD. (2013). Mapa de desarrollo humano de la República Dominicana. PNUD.

Programa de las Naciones Unidas para el Desarrollo, República Dominicana - PNUD-RD. (2019). Desarrollo humano provincial IDHp de República Dominicana. PNUD.

Ramas Arauz, F. E. (2015). Modelos de educación a distancia y rol docente. En F. E. Ramas Arauz (Ed.), Tic en educación: Escenarios y experiencias (pp. 47-84). Ediciones Díaz de Santos.

Scagnoli, N. (2009). A review of online learning and its evolution in Latin America. Policy Futures in Education, 7(5), 555-565. https://doi.org/10.2304/pfie.2009.7.5.555

Tait, A. y O’Rourke, J. (2014). Internationalization and concepts of social justice: What is to be done? En O. Zawacki-Richter y T. Anderson (Eds.), Online distance education: Towards a research agenda. (pp. 75-108). AU Press. https://doi.org/10.15215/aupress/9781927356623.01

UNESCO. (2020a). Interrupción educativa y respuesta al Covid-19. UNESCO.

UNESCO. (2020b). Coalición Mundial para la Educación Covid-19. UNESCO.

UNESCO-IESALC. (2020). Acciones de las redes de educación superior ante el Covid-19. UNESCO-IESALC.

Viñals, A. y Cuenca, J. (2016). El rol del docente en la era digital. Revista Interuniversitaria de Formación del Profesorado, 30(2), 103-114.

Wright, C. R., Dhanarajan, G. y Reju, S. A. (2009). Recurring issues encountered by distance educators in developing and emerging nations. The International Review of Research in Open and Distributed Learning, 10(1), 1-25. https://doi.org/10.19173/irrodl.v10i1.608

\section{Breve CV de los/as autores/as}

\section{Leandro M. Santos}

Profesor invitado de alta calificación (PIAC) del Instituto Superior de Formación Docente Salomé Ureña (ISFODOSU). Biólogo, magíster y doctor en Ciencias Biológicas. Su trayectoria en investigación se centra en estudios de taxonomía, sistemática y biología de abejas de la región Neotropical y aspectos metodológicos y filosóficos de la Zoología. Es autor de capítulo de libro y artículos científicos relacionados con el área. Actualmente es miembro del Grupo de Investigación Interdisciplinario de Investigación Educativa Urania Montás y miembro de la Carrera Nacional de Investigadores en Ciencia, Tecnología e Innovación del MESCyT, República Dominicana.Email: leandro.mattos@isfodosu.edu.do

ORCID ID: https://orcid.org/0000-0002-7126-4420

\section{Diana Grisales}

Bióloga, Magíster y Doctora en Ciencias Biológicas. Actualmente Profesora Invitada de Alta Calificación (PIAC) en el Instituto Superior de Formación Docente Salomé Ureña (ISFODOSU) en República Dominicana. Su trayectoria en investigación se centra en estudios entomológicos en taxonomía, sistemática y biogeografía en la región Neotropical. Es autora de diversos artículos científicos relacionados con su área 
de investigación. Actualmente es miembro del Grupo de Investigación Interdisciplinario de Investigación Educativa Urania Montás del ISFODOSU y miembro de la Carrera Nacional de Investigadores en Ciencia, Tecnología e Innovación del MESCyT, República Dominicana. Email: diana.grisales@isfodosu.edu.do

ORCID ID: https://orcid.org/0000-0002-7313-6035

\section{José Suero Rico}

Profesor y Magíster en Matemáticas. Actualmente, profesor contratado del Instituto Superior de Formación Docente Salomé Ureña (ISFODOSU) en República Dominicana. Sus intereses en investigación están centrados en la Teoría de Grupos, Teoría de Anillos, Teoría de Números, Criptografía y Resolución de Problemas. Actualmente es miembro del Grupo de Investigación Interdisciplinario de Investigación Educativa Urania Montás del ISFODOSU. Email: jose.suero@isfodosu.edu.do

ORCID ID: https://orcid.org/0000-0002-1906-3517 